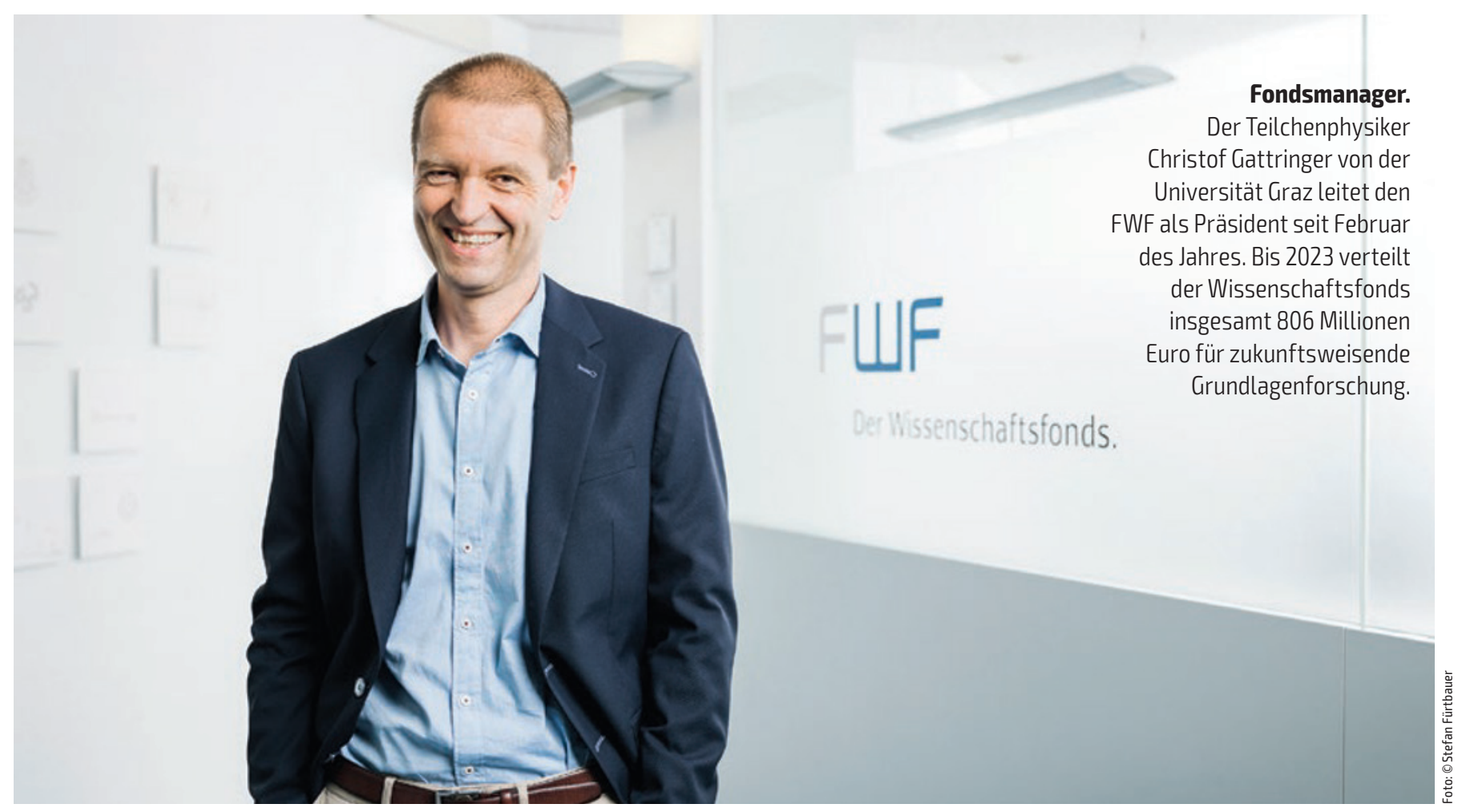

\title{
Forschung gegen das Virus
}

\author{
Der Wissenschaftsfonds FWF hat im Sommer vierzehn neue Projekte zur Corona-Forschung \\ im Umfang von fünf Millionen Euro bewilligt. Die Bandbreite reicht von klinischen und \\ medizinischen Studien bis hin zu Analysen der gesellschaftlichen Auswirkungen der Pandemie. \\ Von Christian F. Freisleben \& Norbert Peter
}

Delta macht der Welt zu schaffen. Die Virusvariante sorgt dafür, dass der Kampf gegen die Pandemie noch lange nicht ausgestanden scheint. Eine wichtige Rolle zur Abwehr des Virus spielt die Pandemieforschung: Das Wissen um COVID-19 liefert die Werkzeuge, um dem Erreger den Schrecken zu nehmen. In einer Kuratoriumssitzung des FWF im Juli wurden 14 neue Forschungsprojekte rund um das Corona-Virus auf Schiene gebracht. Das Fördervolumen der neuen Projekte beträgt fünf Millionen Euro.

Die 14 Vorhaben sind Bestandteil eines Programms, das der FWF als Reaktion auf die Corona-Pandemie aufgesetzt hat und das in einem Schnellverfahren beschleunigt umgesetzt wird. Die Resonanz in der Forschungscommunity ist hoch: Bislang reichten 268 Forschende aus ganz Österreich Projekte und Studien ein. Davon konnten - ohne die Juli-Projekte - „insgesamt 40 Projekte mit einem Fördervolumen von 13 Millionen Euro“, erklärt Christof Gattringer, Präsident des FWF. Die Themenbreite der Projekte reicht von klinischen und medizinischen Studien bis hin zu Analysen der gesellschaftlichen Auswirkungen der Pandemie. Der FWF-Präsident betont den Stellenwert der Grundlagenforschung: „Die schnelle Entwicklung der Vakzine ist ein Triumph der Wissenschaft. Das wäre ohne die jahrzehntelange Grundlagenforschung nicht möglich gewesen. "Corona zeige, wie wichtig es ist, als Gesellschaft auf eine breite Basis an Kompetenzen und auf exzellente Forschende vertrauen zu können. Forschende verpflichten sich bei FWF-Anträgen dem Prinzip des Open Source: Sie legen die Endergebnisse ohne Bezahlschranken offen und dokumentieren auch die Methoden, die sie einsetzen. „Wir sichern den schnellen Wissenstransfer zwischen den Forschenden, den Forschungsstätten aber auch zwischen Wissenschaft, Wirtschaft und Gesellschaft“, betont Gattringer.

\section{Wissenschaftsfonds erreicht Forschende}

Georg Langs vom Computational Imaging Research Lab der Universitätsklinik für Radiologie und Nuklearmedizin beschäftigt sich in seinem Projekt mit Möglichkeiten des Machine Learning, um mit den großen Datenmengen rund um Pandemien umgehen zu können. Langs unterstreicht, wieviel „die Forschung betreffend Diagnose, Impfung oder Behandlung bei der Bewältigung der Corona-Pandemie in einer bemerkenswert kurzen Zeit geleistet hat“. Langs' Projekt entwickelt neue Methoden im Bereich des maschinellen Lernens für die medizinische Bildgebung. „Wir wollen neue Krankheitsbilder oder Varianten bestehender Krankheiten rechtzeitig entdecken und damit rasche Reaktionen ermöglichen." Das zweite Ziel von Langs' Forschungsarbeiten ist es, in einer ers- 
ten Phase einer Pandemie möglichst rasch Vorhersagemodelle zur Verfügung zu stellen. Basis der Prognosetools sind Beobachtungen, die in der klinischen Routine - Diagnostik und Behandlung - gemacht werden.

Dass das Forschungsfeld sehr weit gefasst ist, sieht man an den genehmigten Projekten. Es sind bei Weitem nicht nur Forschende aus der Medizin beteiligt. Tatiana Konrad vom Institut für Anglistik und Amerikanistik an der Universität Wien leitet zum Beispiel ein Projekt, das die Pandemie aus der Perspektive des Umgangs mit Umweltverschmutzung betrachtet: Luftverschmutzung wurde in vielen Regionen als möglicher, wesentlicher Faktor der stärkeren Verbreitung für das Virus identifiziert. Luft, Gesundheit und Krankheit werden in diesem transdisziplinären Projekt auf neue Weise gemeinsam betrachtet und auch so sollen Beiträge zu präventiven Maßnahmen geliefert werden.

Auch das Projekt von Ina Bergheim vom Department für Ernährungswissenschaften der Universität Wien sucht Wege, die Auswirkungen des Virus einzudämmen: „Unsere Arbeit soll dazu

\section{DIE GUTEN IM TÖPFCHEN}

- COVID und Bipolare Störungen. Die Fachärztin für Psychiatrie und Molekularbiologie Susanne Bengesser von der Medizinischen Universität Graz beschäftigt sich mit der Situation von Menschen mit bipolaren Störungen und genetischen Risiken für eine COVID-19-Infektion.

- Zucker gegen COVID. Peter Hinterdorfer vom Institut für Biophysik an der Johannes Kepler Universität Linz und Miriam Klausberger, Institut für Molekulare Biotechnologie der Universität für Bodenkultur in Wien, forschen gemeinsam zur Fähigkeit des SARS-CoV-2-Virus, in Zellen einzudringen und sich dort zu vermehren. Erkenntnisse über den Einfluss unterschiedlicher Zuckermoleküle sollen helfen, Therapien zu entwickeln.

- COVID und Diabetes. Julia Kerschbaum von der Klinik für Innere Medizin an der Universität Innsbruck geht in ihrer Forschung von vermuteten hohen Mortalitätszahlen bei Menschen mit Diabetes und einer COVID-19-Infektion aus, wobei besonders Personen betroffen sein könnten, die sich regelmäßig einer Dialyse unterziehen müssen.

- Pillen gegen COVID. Dorothee von Laer vom Institut für Virologie der Medizinische Universität Innsbruck analysiert mit ihrer Forschungsgruppe Details zur Rolle von Auswirkungen von Medikamenten auf Proteasen, um die Vermehrung von Corona-Viren zu stoppen.

- COVID und Enzyme. Eduard Stefan vom Institut für Biochemie der Universität Innsbruck analysiert mit seinem Team, inwieweit Kinasen - Enzyme, die normalerweise bei der Krebserkrankung eine Rolle spielen, - bei der Bekämpfung von COVID-19 zum Einsatz kommen könnten. beitragen, molekulare Mechanismen besser zu verstehen, die den Entzündungsprozessen und der Gewebeschädigung des SARSCoV-2-Virus unterliegen." Darauf basierende präventive Maßnahmen sollen beitragen, besser auf neue Infektionswellen vorbereitet zu sein. Im Zentrum der Forschung steht die potenzielle Schutzwirkung von Xanthohumol, einem Polyphenol, das im Hopfen zu finden ist und mit der Nahrung aufgenommen wird. Es soll dem Körper helfen, besser mit einer COVID-19-Infektion umzugehen.

Viele Wissenschaftler haben durch die Pandemie zu neuen Betrachtungsweisen und Interpretationen eigener und fremder Forschungsarbeiten gefunden. Eine Vielzahl neuer Projekte und Veröffentlichungen wurde angestoßen. Die zusätzlichen Mittel staatlicher und privater Investoren haben das Forschungstempo beflügelt. „Ich freue mich, dass durch den FWF Forschung auf eine so schnelle Weise gefördert wird. Dies eröffnet neue Forschungsmöglichkeiten sowie neue Formen der Zusammenarbeit." Bergheim hofft, dass die Vielzahl an Fördermöglichkeiten im Kontext mit „Corona“ nicht zu Lasten anderer Bereiche ging, "da diese ja durch ,Corona nicht weniger relevant wurden, sondern nur temporär teilweise in den Hintergrund des Interesses getreten sind.“

\section{Forschen für die Zeit danach}

Tatsächlich wirken die Resultate aus der unmittelbaren CoronaForschung weit über den ursprünglichen Fokus hinaus. Ein Beispiel dafür liefert Robert Strassl mit seinem Team in der Abteilung für klinische Virologie der Medizinischen Universität Wien: Er will schnellere Tests entwickeln. Mittels eines elektronischen Biosensors soll dann rascher feststehen, welche Virusvariante der Patient hat und ob er noch ansteckend ist. Das System kann dann auch für andere Viren adaptiert werden und seinen Wert auch in der Zukunft unter Beweis stellen.

Dass es eine Zeit danach gibt, weiß man auch im FWF. Gefragt danach, wo er Österreich in fünf Jahren in Bezug auf die Pandemie sieht, meint Christof Gattringer: „Ich denke, wir werden uns daran gewöhnt haben, sie wird aber nicht mehr unseren Alltag dominieren, wir werden Corona insgesamt unter Kontrolle haben." Somit könnten wir uns als Gesellschaft den anderen großen Herausforderungen wie der Klimakrise zuwenden. Die Wissenschaft zeige, wie dringend hier der Handlungsbedarf ist: „Das wird sich in fünf Jahren weiter zuspitzen, wenn wir nicht gegensteuern“, so Gattringer. // 